\title{
Publisher Correction: Dissociable roles of cortical excitation-inhibition balance during patch-leaving versus value-guided decisions
}

\author{
Luca F. Kaiser (1D, Theo O. J. Gruendler (1), Oliver Speck, Lennart Luettgau \& Gerhard Jocham (1)
}

Correction to: Nature Communications https://doi.org/10.1038/S41467-020-20875-W, published online 10 February 2021.

The original version of this Article contained an error in the "Methods" section, under the "Decision-making task" heading. The results of a $t$ test were reported with the incorrect degrees of freedom, incorrectly written as "t18858 $=-20.915, p<0.001$." The correct version replaced "t18858" with "t18558." This has been corrected in both the PDF and HTML versions of the Article.

The original version of this Article also contained an error in the "Methods" section titled "Behavioural modelling of value-guided decisions" in which Eqs. 5 and 6 inadvertently swapped during production. The incorrect version stated

Since it is known that humans do not weigh magnitudes and probabilities in a statistically optimal way, we considered systematic distortions in the weighting of reward information in our models $(u(m)$ and $w(p)$, for reward magnitudes and probabilities, Eqs. 5 and 6 , respectively) ${ }^{56}$.

$$
u\left(m_{\mathrm{O}}\right)=m_{\mathrm{O}}{ }^{\alpha}
$$

where $p_{\mathrm{O}}$ are the objective reward probabilities and $\gamma$ is a free parameter used to fit subjective reward probabilities. Subjective magnitudes were estimated by:

$$
w\left(p_{\mathrm{O}}\right)=\frac{p_{\mathrm{O}}^{\gamma}}{\left(p_{\mathrm{O}}^{\gamma}+\left(1-p_{\mathrm{O}}\right)^{\gamma}\right)^{1 / \gamma}}
$$

The correct version states

Since it is known that humans do not weigh magnitudes and probabilities in a statistically optimal way, we considered systematic distortions in the weighting of reward information in our models $\left(w\left(p_{\mathrm{O}}\right)\right.$ and $u\left(m_{\mathrm{O}}\right)$ for reward probabilities and magnitudes, Eqs. 5 and 6 , respectively $)^{56}$.

$$
w\left(p_{\mathrm{O}}\right)=\frac{p_{\mathrm{O}}^{\gamma}}{\left(p_{\mathrm{O}}^{\gamma}+\left(1-p_{\mathrm{O}}\right)^{\gamma}\right)^{1 / \gamma}}
$$

where $p_{\mathrm{O}}$ are the objective reward probabilities and $\gamma$ is a free parameter used to fit subjective reward probabilities. Subjective magnitudes were estimated by:

$$
u\left(m_{\mathrm{O}}\right)=m_{\mathrm{O}}{ }^{\alpha}
$$

The original version of this Article also contained an error in the Methods section under the heading "MRS data acquisition", in which the terms "rM1 and IM1" were incorrectly ordered. The incorrect version stated

Average M1 voxel centroids in standard space were estimated at MNI $x=-28.97 \pm 0.82, y=-18.48 \pm 0.92, z=51.86 \pm 0.59$ and $\mathrm{MNI}$ $x=31.90 \pm 0.71, y=-14.76 \pm 1.06, z=49.76 \pm 0.88$ for $\mathrm{rM1}$ and $1 \mathrm{M} 1$, respectively. 
The correct version states

Average M1 voxel centroids in standard space were estimated at MNI $x=-28.97 \pm 0.82, y=-18.48 \pm 0.92, z=51.86 \pm 0.59$ and MNI $x=31.90 \pm 0.71, y=-14.76 \pm 1.06, z=49.76 \pm 0.88$ for $1 \mathrm{M} 1$ and $\mathrm{rM1}$, respectively.

Published online: 17 June 2021

(c) (1) Open Access This article is licensed under a Creative Commons Attribution 4.0 International License, which permits use, sharing, adaptation, distribution and reproduction in any medium or format, as long as you give appropriate credit to the original author(s) and the source, provide a link to the Creative Commons license, and indicate if changes were made. The images or other third party material in this article are included in the article's Creative Commons license, unless indicated otherwise in a credit line to the material. If material is not included in the article's Creative Commons license and your intended use is not permitted by statutory regulation or exceeds the permitted use, you will need to obtain permission directly from the copyright holder. To view a copy of this license, visit http://creativecommons.org/licenses/by/4.0/.

(c) The Author(s) 2021 